\title{
MUSHROOM POISONING INDUCED ACUTE LIVER FAILURE WITH DERANGED COAGULATION PROFILE
}

\author{
Anupam Dutta1, Pranjal Kumar Dutta², Swaroop Kar $^{3}$ \\ ${ }^{1}$ Assistant Professor, Department of Medicine, Assam Medical College and Hospital, Assam. \\ ${ }^{2}$ Assistant Professor, Department of Medicine, Assam Medical College and Hospital, Assam. \\ ${ }^{3}$ Registrar, Department of Medicine, Assam Medical College and Hospital, Assam.
}

\begin{tabular}{l}
\hline ABSTRACT \\
BACKGROUND \\
Mushroom poisoning cases are frequently reported from Upper Assam during the monsoon season with varying but high mortality. \\
Most of the cases reported from upper Assam are amatoxin poisoning. In one of our previous publications we have concluded that \\
raised serum bilirubin, unconjugated bilirubin, aspartate aminotransferase (AST) and alanine aminotransferase (ALT) were \\
predictors of mortality. In this case series, we report 14 cases that came in the early spring of 2017 and we found that raised \\
prothrombin time was a strong indicator of mortality in patients with mushroom poisonings.
\end{tabular}

\section{MATERIALS AND METHODS}

All patients who came to our Institute in 2017 with alleged history of mushroom poisoning were included in our study. A detailed history was taken and proper clinical examinations were done. Biochemical tests were done and correlated with patient's outcome. All patients were treated according to our Institutional protocol.

\section{RESULTS}

Fourteen (14) adults were included in the series with eight (8) males and six (6) females. Four (4) patients expired and ten (10) survived. The patients who died had significant high liver enzymes. They also had significantly higher prothrombin time (mostly above 50 seconds), whereas in those patients who survived their average prothrombin time was 15.9 seconds initially (on day two of ingestion of mushroom) and 11.7 seconds during recovery (day eight of ingestion of mushroom).

\section{CONCLUSION}

We conclude that high Prothrombin time, especially more than 50 seconds, is associated with high mortality in patients with mushroom poisoning.

\section{KEYWORDS}

Mushroom Poisoning, Prothrombin Time, Acute Liver Failure, Aspartate Aminotransferase (AST), Alanine Aminotransferase (ALT).

HOW TO CITE THIS ARTICLE: Dutta A, Dutta PK, Kar S. Mushroom poisoning induced acute liver failure with deranged coagulation profile. J. Evolution Med. Dent. Sci. 2017;6(66):4783-4786, DOI: 10.14260/Jemds/2017/1036

\section{BACKGROUND}

Mushroom poisoning cases are frequently reported from Upper Assam during the monsoon season. Their reported fatalities range from $43.75 \%(21 / 48)^{1}$ to $14.7 \%(10 / 68) .^{2}$ Out of 5000 species of mushroom, only 100 are poisonous and 32 species have been associated with fatalities. ${ }^{3}$ More than $90 \%$ of cases of fatal poisoning are caused by Amanita phalloides (Death cap) or Amanita verna (Destroying angel). Most of the cases reported from upper Assam are amatoxin poisoning. Alpha-Amatoxin is thermostable, can resist drying for years and is not inactivated by cooking. Rapidly absorbed through the gastrointestinal tract, the amatoxin reaches hepatocytes through the enterohepatic circulation and inhibits production of messenger RNA and protein synthesis leading in turn to cell necrosis.

Financial or Other, Competing Interest: None.

Submission 10-07-2017, Peer Review 07-08-2017,

Acceptance 12-08-2017, Published 17-08-2017.

Corresponding Author:

Dr. Anupam Dutta,

REVTI House,

Purnananda Road,

Shantipara,

Dibrugarh- 786001, Assam

E-mail: dranupamdutta80@gmail.com

DOI: $10.14260 /$ jemds $/ 2017 / 1036$

\section{(c) $(1)$}

The severity of mushroom poisoning usually depends on the type of mushroom, amount of mushroom ingested, preexisting hepatic disease and a host of other factors. Ganzert et $\mathrm{al}^{4}$ retrospectively analysed the outcome of a large series of amatoxin intoxication cases and found that predictors of death were the prothrombin time in combination with the serum creatinine level on 3 - 10 days after ingestion. In one of our previous publications we have concluded that raised serum bilirubin, unconjugated bilirubin, aspartate aminotransferase (AST) and alanine aminotransferase (ALT) were predictors of mortality in patients with mushroom poisoning. ${ }^{5}$ Here, we are reporting a case series of 14 patients with acute liver failure following mushroom poisoning that presented to our hospital in the early spring of 2017 with raised prothrombin time.

\section{MATERIALS AND METHODS}

All patients who came to our Institute in the spring of 2017 with alleged history of Mushroom poisoning were included in our study. A detailed history was taken and proper clinical examinations were done. Biochemical tests were done and correlated with patient's outcome. All patients were treated according to our Institutional protocol, which included early hydration with crystalloids (mostly Ringer's Lactate and Normal Saline), Ryle's tube aspiration, Silymarin, Nacetylcysteine, fresh frozen plasma, vitamin $\mathrm{C}$ and other supportive treatment. 
For prothrombin time, blood was collected in a test tube containing liquid sodium citrate, which after mixing is centrifuged. A sample of plasma is extracted and excess of calcium is added to reverse the effect of citrate. Then in order to activate the extrinsic/ tissue factor clotting cascade, factor III is added and time for the sample to clot is measured optically. INR (International normalised ratio) is the prothrombin time for the patient's sample divided by the result for controlled plasma.

\section{RESULTS}

Fourteen (14) adults were included in the series with eight (8) males and six (6) females. Four (4) patients expired and ten (10) survived. In the first cohort of 8 cases who were promptly brought to our tertiary care hospital, two cases developed fulminant liver failure and bleeding diathesis and expired. In the second cohort of three patients all survived. In the third cohort of two patients, one had very high liver enzymes and prothrombin time and she died within 24 hours of hospitalisation. The last patient was delayed hospitalisation by more than 100 hours from the time of ingestion. He also died of fulminant hepatic failure. The patients who died had significant high liver enzymes and prothrombin time greater than 50 seconds.

\section{Cohort 1 (8 Cases)}

A family of six (6) which included three females aged 55 years, 42 years, 17 years and three males aged 24 years, 22 years and 17 years had prepared mushroom curry on $7^{\text {th }}$ March 2017 and invited three guests who were 36, 38 and 60 -year-old males from the same village. The host family had collected mushrooms from their backyard (which is partly forest area) and had done similar mushroom collection several times in the past including last year. They had prepared the mushroom in light oil with brinjal and other vegetables in a gravy preparation which they had served with rice, lentil soup (dal), potato fries and pickle. All the patients had commented that the meal was very tasty, especially the mushroom curry. After 24 hours of ingestion of the meal they developed vomiting, pain abdomen and diarrhoea and were admitted to a nearby hospital. During this period, they did not take anything remarkable like alcohol or other oily meals. Next day they were hospitalised after they developed severe dehydration and signs of acute kidney injury. They were promptly referred to our tertiary care centre for further management. They were treated with three to four litres of intravenous crystalloids per 24 hours, mostly Ringer's Lactate and Normal Saline. They were also given nasogastric (Ryles) tube and kept nil orally for 48 to 72 hours. Regular aspiration of Ryles tube was done to remove any bilious material. They were restarted with small oral liquid diets after 36 to 48 hours if liver function test and renal function test improved. They also received 15 to $20 \mathrm{~mL}$ of Silymarin syrup thrice daily by Ryles tube; intravenous $\mathrm{N}$-acetylcysteine was given as a loading dose of $100 \mathrm{mg} / \mathrm{kg}$ over 6 hours followed by $6.25 \mathrm{mg} / \mathrm{kg} / \mathrm{hr}$ and continued for 48 to $72 \mathrm{hrs}$. or till improvement. Vitamin $\mathrm{C}$ tablets were crushed and given via Ryles tube diluted with water thrice daily and oral Vitamin C tablets were added when the patient was allowed to take orally. Two patients, one 42-year-old female and another 60-year-old male, whose prothrombin time were not recordable in first reporting eventually died. Their liver enzymes were also raised more than hundred times the normal range (Table 1). They had developed hepatic encephalopathy and bleeding diathesis in their final hours. In rest of the patients the prothrombin time and liver enzymes were raised, but they responded to treatment and came down within a week's time. One person who was a 36-year-old male guest in the mushroom meal was not referred to our hospital at that time.

\section{Cohort 2 (3 Cases)}

Three female tea garden labourers aged 45, 27 and 16 years came to our tertiary care centre after being referred from primary care centre for pain abdomen, diarrhoea and vomiting following ingestion of wild mushrooms from garden backyard. They had done similar mushroom hunting in the past, but never encountered such symptoms. They had boiled the mushroom with spinach and other vegetables and ate with rice and pickle. During their initial assessment, they were initially dehydrated and their liver enzymes and prothrombin time were raised. They were adequately rehydrated and given the standard of care according to our hospital protocol. They recovered in ten days' time and were discharged. They came for followup after two weeks and their parameters were back to normal.

\section{Case 3 ( 2 Cases)}

A 45-year-old female and her 18-year-old son were admitted in our institute in a state of altered consciousness following several episodes of diarrhoea and vomiting. On taking a detailed history, they told us about ingestion of wild mushrooms which she had taken from forest area and prepared for herself and her son. This was her first time and she said that she was attracted by the size and bright colour of the mushroom. She had previously ingested mushroom collected by other villagers, but never collected herself. The mushroom was cooked in oil with potato, onion and brinjal. They also took country liquor along with mushroom. They were initially admitted in a peripheral hospital and were referred to our centre after 48 hours. She was severely dehydrated and her serum creatinine level was also raised $(3.6 \mathrm{mg} / \mathrm{dL})$. Her enzymes were raised more than hundred folds and prothrombin time was 57 . She was treated with fluids, Silymarin, fresh frozen plasma but she developed acute liver failure and hepatic encephalopathy. She developed bleeding diathesis and petechiae all over her body. She was given two units of fresh frozen plasma, but she died within next 24 hours. Her son recovered with our standard treatment protocol over a period of one week's time and was discharged after 6 days. He came for followup after two weeks and was found to be completely recovered both clinically and biochemically.

\section{Case 4 (1 Case)}

This 36-year-old male patient had ingested cooked mushroom with the first cohort, but was brought to our hospital four (4) days after the previous cohort of patients. So there was a delay of proper treatment by more than 100 hours (Image 1) and by the time he came to us he had already developed acute liver injury and grade III hepatic encephalopathy. His AST was 6857 IU and ALT was 4543 IU. His Prothrombin time showed that blood was not clotting. He was admitted in the ICU and was treated with several bottles of fresh frozen plasma. After 36 hours, he was intubated and put on assisted ventilation. He died within 48 hours of hospitalisation. 


\begin{tabular}{|c|c|c|c|c|c|c|c|c|c|c|c|}
\hline & \multicolumn{4}{|c|}{ AST (IU) } & \multicolumn{4}{|c|}{ ALT (IU) } & \multicolumn{3}{|c|}{ PT (Sec) } \\
\hline Age/Sex & D 2 & D 4 & D 6 & D 8 & D 2 & D 4 & D 6 & D 8 & D 2 & D 6 & D 8 \\
\hline $24 \mathrm{M}$ & 185 & 32 & 29 & 27 & 104 & 40 & 34 & 31 & 13.4 & 11.9 & 10.8 \\
\hline $22 \mathrm{M}$ & 232 & 106 & 82 & 47 & 231 & 863 & 714 & 301 & 12.7 & 10.9 & 10.7 \\
\hline $17 \mathrm{M}$ & 68 & 69 & 44 & 39 & 57 & 412 & 293 & 227 & 14.4 & 12.7 & 11.2 \\
\hline $55 \mathrm{~F}$ & 179 & 91 & 63 & 54 & 194 & 60 & 86 & 48 & 16.7 & 12.4 & 12.3 \\
\hline $42 \mathrm{~F}$ & 669 & 10917 & - & - & 377 & 7720 & - & - & $\mathrm{NC}$ & $\mathrm{NC}$ & - \\
\hline $17 \mathrm{~F}$ & 107 & 2124 & 394 & 214 & 89 & 2953 & 2408 & 1725 & 16.8 & 13.2 & 12.8 \\
\hline $38 \mathrm{M}$ & 115 & 697 & 292 & 114 & 996 & 1498 & 839 & 457 & 19.2 & 16.7 & 14.3 \\
\hline $60 \mathrm{M}$ & 799 & 5923 & - & - & 548 & 4442 & - & - & $\mathrm{NC}$ & 40 & - \\
\hline $45 \mathrm{~F}$ & 384 & 189 & 114 & 76 & 352 & 110 & 78 & 45 & 17.8 & 14.3 & 10.4 \\
\hline $27 \mathrm{~F}$ & 228 & 108 & 84 & 42 & 185 & 129 & 94 & 68 & 15.3 & 13.7 & 11.3 \\
\hline $16 \mathrm{~F}$ & 187 & 78 & 57 & 49 & 167 & 89 & 64 & 57 & 15.9 & 13.5 & 10.7 \\
\hline $45 \mathrm{~F}$ & 4730 & - & - & - & 3827 & - & - & - & 57 & - & - \\
\hline $18 \mathrm{M}$ & 483 & 229 & 198 & 115 & 334 & 286 & 147 & 101 & 16.9 & 13.2 & 12.9 \\
\hline $36 \mathrm{M}$ & 6857 & - & - & - & 4543 & - & - & - & $\mathrm{NC}$ & - & - \\
\hline
\end{tabular}

AST - Aspartate aminotransferase; ALT - Alanine aminotransferase; PT - Prothrombin Time;

D - Day from ingestion; M - Male; F - Female; NC - Not Clotted; IU - International Unit.

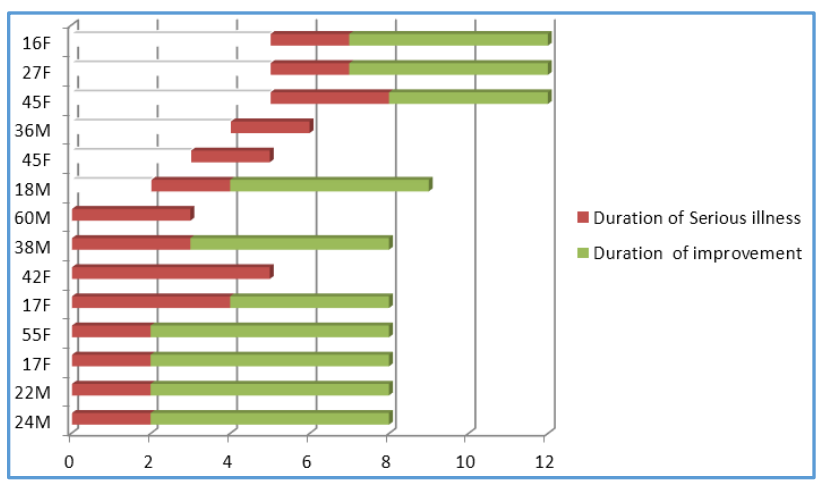

Figure 1. Duration of Serious Illness and Recovery of Patients with Mushroom Poisoning

The patients of mushroom poisoning who had expired, had significantly higher prothrombin time (mostly above 50 seconds), whereas in those patients who survived their average prothrombin time was 15.9 seconds initially (on day two of ingestion) and 11.7 seconds during recovery (day eight of ingestion) (Image 2).

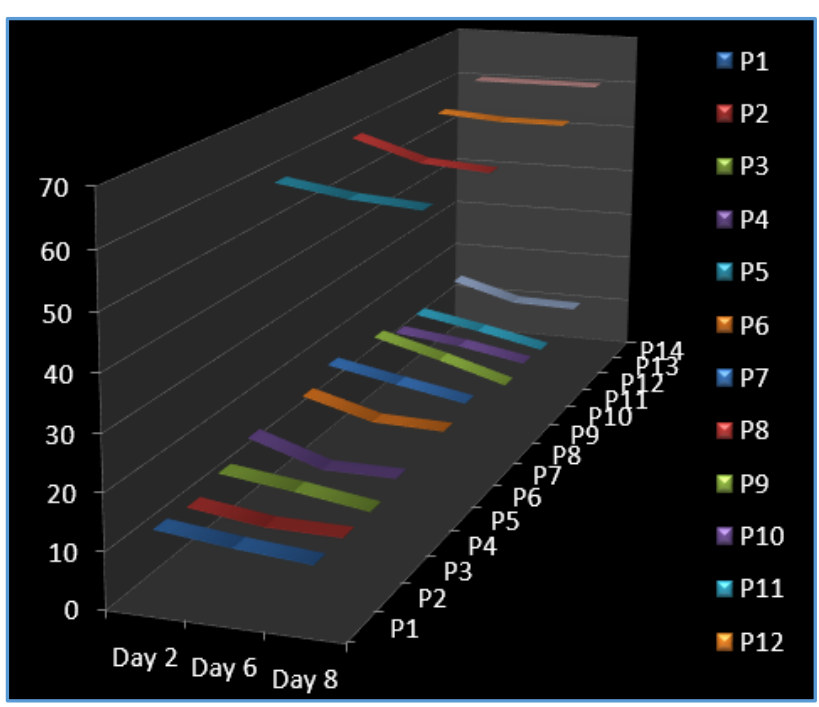

Figure 2. Higher Prothrombin Time correlating with Mortality in Mushroom Poisoning Cases

\section{DISCUSSION}

Das $\mathrm{AK}^{6}$ had described that classically the patient undergoes four (4) stages of symptomatology following mushroom poisoning (Image 3). Patients develop epigastric abdominal pain, vomiting and severe cholera-like diarrhoea that may contain blood and mucous, and typically starts between 6 and 24 hours after mushroom ingestion. Liver enzymes and bilirubin are usually normal. Electrolyte abnormalities consistent with a secretory diarrhoea (e.g. hypokalaemia and metabolic acidosis) may also occur. Profound gastrointestinal fluid losses may lead to dehydration, acute renal failure and circulatory shock. With appropriate supportive care of the delayed gastroenteritis, patients appear to improve between approximately 24 to 36 hours after mushroom consumption. Elevations in the liver enzymes, aspartate aminotransferase (AST) and alanine aminotransferase (ALT) are typically detectable between 24 and 36 hours after mushroom ingestion and typically peak by approximately 60 to 72 hours post-ingestion. In patients with severe poisoning, the patient may go directly from severe gastroenteritis into fulminant organ failure with hyperbilirubinaemia, dramatic elevations in liver enzymes over 1000 to $2000 \mathrm{IU} / \mathrm{L}$, prolongation of the prothrombin time, renal insufficiency and disseminated intravascular coagulopathy. Approximately, two to four days after mushroom consumption, severely poisoned patients develop hepatic failure, often complicated by acute renal failure. Massive simultaneous hepatocyte cell death disrupts hepatic venous and biliary flow. Peak transaminase elevations are typically seen between 48 and 72 hours postingestion and may be greater than 2000 IU/L. Direct nephrotoxic effects are seen in the proximal and distal convoluted renal tubules. Loss of hepatic function over the following days causes hypoglycaemia, coagulopathy, encephalopathy and fluid shifts with progression to multiorgan failure. 


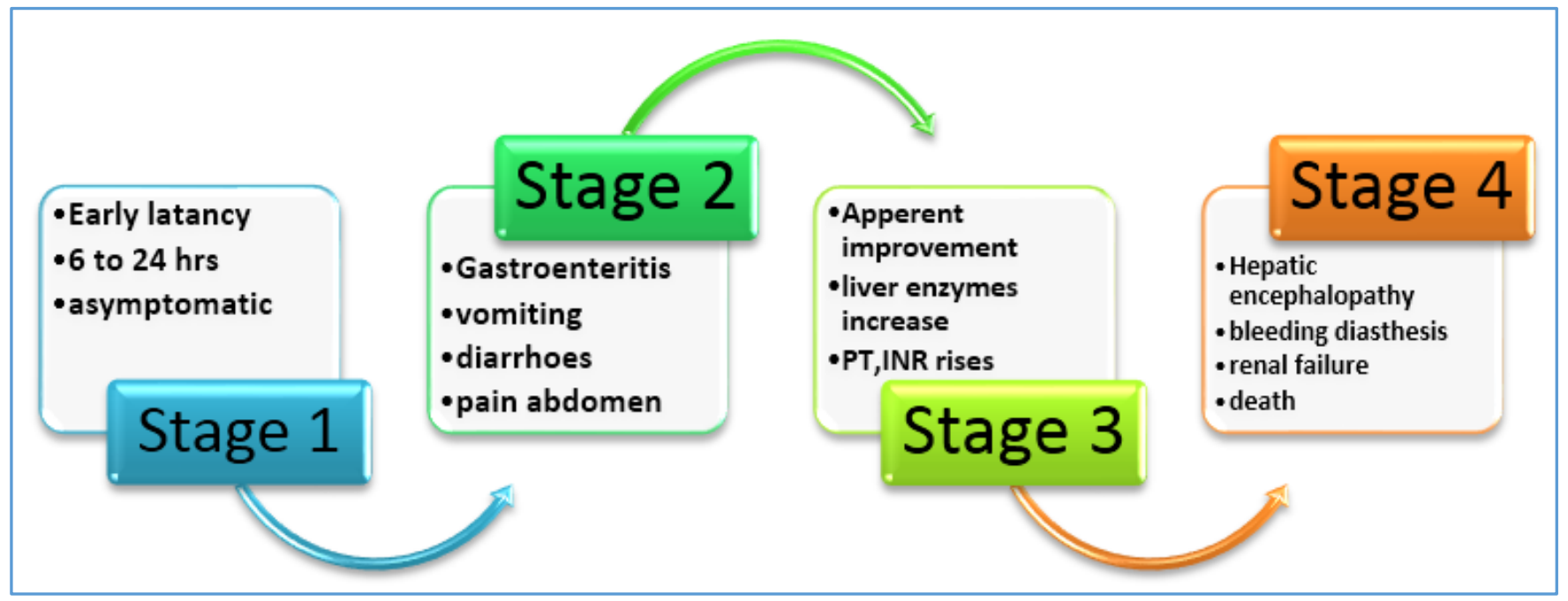

Figure 3. Stages of Mushroom Poisoning

The mortality in mushroom poisoning varies from region to region and is mostly dependent on the type of mushroom ingested, facilities available like liver transplant and critical care management. Though there have been very few mushroom poisonings published in literature from India, most have shown high mortality. Ganzert et $\mathrm{al}^{4}$ retrospectively analysed the outcome of a large series of amatoxin intoxication cases and found that predictors of death were the prothrombin index in combination with the serum creatinine level on 3 - 10 days after ingestion. Passo B et $\mathrm{al}^{7}$ and Teutsch $\mathrm{C}$ et $\mathrm{al},{ }^{8}$ both described that liver transplantation improve mortality in amatoxin-induced acute liver failure in selected patients and before development of grade 4 hepatic encephalopathy. But access to liver transplant is trivial, more so in developing countries. Overall, the prognosis of amanitin-induced acute liver failure remains quite poor, especially in our part of the country.

When conservative management is the only option, early identification of the risk factors of mortality is important to classify patients with mushroom poisoning to high risk and low risk. In one of our earlier paper we have already concluded that raised serum bilirubin, unconjugated bilirubin, aspartate aminotransferase (AST), alanine aminotransferase (ALT) and prothrombin time were predictors of mortality in patients with mushroom poisoning. ${ }^{5}$ In that case series, Aspartate Aminotransferase (AST) $(3549 \pm 150$ vs. $434.7 \pm 100)$ and Alanine Aminotransferase (ALT) $(5549 \pm 100$ vs. $707.5 \pm 110)$ were significantly higher in the group who died due to mushroom poisoning. Highest prothrombin time in the same study was $70.8 \pm 10$ seconds in patients who died following mushroom poisoning and $10.6 \pm 5$ seconds in those who survived. This study also shows a prothrombin time of more than 50 seconds was associated with mortality.

\section{CONCLUSION}

We conclude that high prothrombin time, especially more than 50 seconds is associated with high mortality in patients with mushroom poisoning.

\section{REFERENCES}

[1] Dutta A, Kalita BC, Pegu AK. A study of clinical profile and treatment outcome of mushroom poisoning-a hospital based study. Assam Journal of Internal Medicine 2013;3(2):13-7.

[2] Sharma J, Malakar M, Sandiguria E, et al. An expressive study of mushroom poisoning cases in Lakhimpur district of Assam. International Journal of Advancements in Research \& Tecnology 2013;2(9):828.

[3] Lsewis JH. Sleisenger and Fordtran's Gastrointestinal and Liver disease. Chapter 89. 10 $0^{\text {th }}$ edn. USA: Elsevier 2016.

[4] Ganzert M, Felgenhauer N, Zilker T. Indication of liver transplantation following amatoxin intoxication. J Hepatol 2005;42(2):202-9.

[5] Dutta A, Bora K. Elevated transaminases as predictor of mortality in mushroom poisoning patients. Journal of Evolution of Research in Medical Biochemistry 2016;2(2):1-3.

[6] Das AK. Mushroom poisoning-need for our awareness and a treatment protocol. Assam Journal of Internal Medicine 2013;3(2):4-12.

[7] Passo B, Harrison DC. A new look at an old problem: mushroom poisoning. Clinical presentations and new therapeutic approaches. Am J Med 1975;58(4):505-9.

[8] Teutsch C, Brennan RW. Amanita mushroom poisoning with recovery from coma: a case report. Ann Neurol 1978;3(2):177-9. 